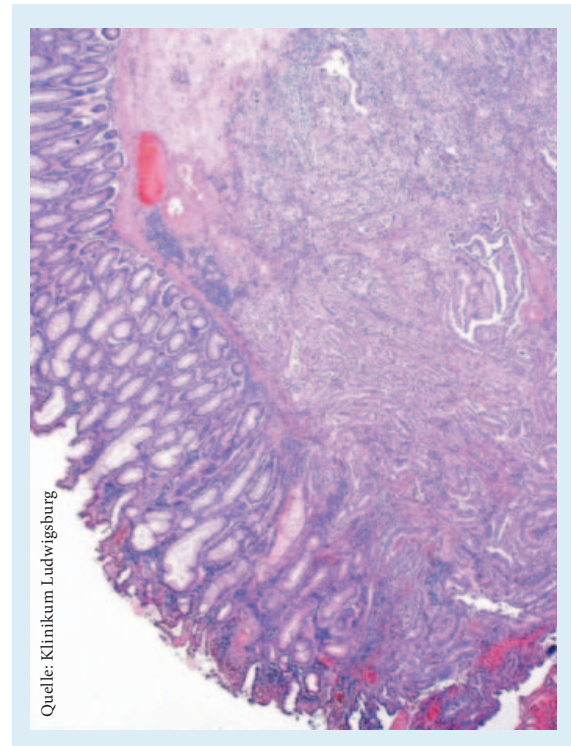

Magen-Darm-Erkrankungen

Entscheidungen mit dem Kopf zu treffen, gilt als klug, sie mit dem Bauch zu begründen als wenig sinnvoll. Auf Seite 73 erfahren Sie, dass Wissenschafter mittlerweile von einem Bauchhirn sprechen. Der Darm ist ein stark mit Nerven versorgtes Organ, das nicht nur Befehle empfängt, sondern auch seinen Beitrag leistet, den Rest des Körpers zu steuern. Er entscheidet über das Wohlsein und das Krankfühlen. Und was zwei Experten der Ganzheitsmedizin zur Behandlung solcher Beschwerden in der täglichen medizinischen Praxis empfehlen, lesen Sie in unserem Expertengespräch auf Seite 75. Und Ärzte, die bei Morbus Crohn, Colitis ulcerosa und Reizdarm auf die Komplementärmedizin setzen, decken damit ein Bedürfnis ihrer Patienten, was auf Seite 80 steht.

\title{
Aus dem Bauch heraus
}

Probleme schlagen auf den Magen. Unser Bauch wird meistens als Schlachtfeld dessen wahrgenommen, was den Kopf beschäftigt. Dabei ist es eher umgekehrt: Der Bauch bestimmt den Kopf und füttert inn mit einer Fülle von Informationen. Denn im Magen-Darm-Trakt sitzt ein zweites «Hirn». Dank dieser Erkenntnisse sind alte Ansätze der Ganzheitsmedizin wieder brandaktuell.

Vor der alles entscheidenden Kür, in der die Schweizerin Sarah Meier ihren Titel als Europameisterin im Eiskunstlauf holte, war die 26-Jährige «fürchterlich nervös». Fast mantraartig wiederholte die Eiskunstläuferin diese Worte nach dem Wettkampf. Was sie auch immer wieder sagte: «Die Nervosität hat mir auf den Magen geschlagen.» Ihrer Leistung zumindest vermochte dies nichts anzuhaben - Sarah Meier lief in Bern die Kür ihres Lebens.

Harry Müller ist Trader in einem internationalen Finanzinstitut, das mit Rohstoffen handelt. Er ist sehr erfolgreich: Ob Öl, Gold, Silber oder Aluminium, stets trifft er den richtigen Zeitpunkt für Kauf und Verkauf. Von bewundernden Kollegen nach seinem Erfolgsrezept gefragt, antwortet Müller jeweils knapp: «Alles Bauchgefühl.»

Den Zusammenhang zwischen dem Befinden und dem Magen-DarmTrakt spiegelt die Sprache in zahlreichen Redewendungen. Im Bauch haben wir je nach Gefühl und Situation eine Wut oder ein flaues Gefühl, Verliebte eher ein Flattern oder gar Schmetterlinge. Soll die Liebe von Dauer sein, geht sie am besten durch den Magen; dann hat man den Liebsten zum Fressen gern. Ein schönes Gefühl ist es auch, aus dem Bauch heraus leben und handeln zu können. Negatives schluckt man unter Umständen hinunter, frisst es in sich hinein oder man kaut darauf herum.
Wenn etwas ganz schlimm ist, finden wir es zum Kotzen. Ein schlechtes Erlebnis liegt uns schwer im Magen, und wir haben Mühe, es zu verdauen. Manchmal ist uns auch ganz flau im Magen, es dreht sich uns der Magen um, es stösst etwas sauer auf, es vergeht uns der Appetit oder wir haben es gründlich satt. Der Appetit kann aber auch gesegnet sein.

Die vielfältigen Beziehungen zwischen körperlichem und psychischem Befinden und wie sie sich sprachlich äussern, kommen nicht von ungefähr. «Diese Beziehungen haben ein grosses Ausmass von Empirie in sich», sagt Reinhard Saller, Professor für Naturheilkunde an der Universität Zürich. «Der Magen-Darm-Trakt ist ein Feld, auf dem sich Leib und Seele treffen. Und es ist ganz klar, dass der Darm ganz erheblich unsere Stimmung, unsere Emotionalität, aber auch unser Denken und unsere Erinnerungen, also unsere ganzen kognitiven Fähigkeiten, beeinflussen kann.»

Meistens allerdings wird der Weg eher in umgekehrter Richtung wahrgenommen: dass das, was Kopf und Seele umtreibt, sich auf den MagenDarm-Trakt auswirkt. Tatsächlich beobachten Ärzte und Psychosomatiker eine Zunahme von Magen-DarmBeschwerden, die sie auf die zunehmende Hektik im Beruf und Privatleben, auf den allgemeinen «Stress", um das Schlagwort zu bemühen, zurückführen. Vor allem für funktionelle Syndrome wie Reizmagen und Reiz-

\section{KARGER}




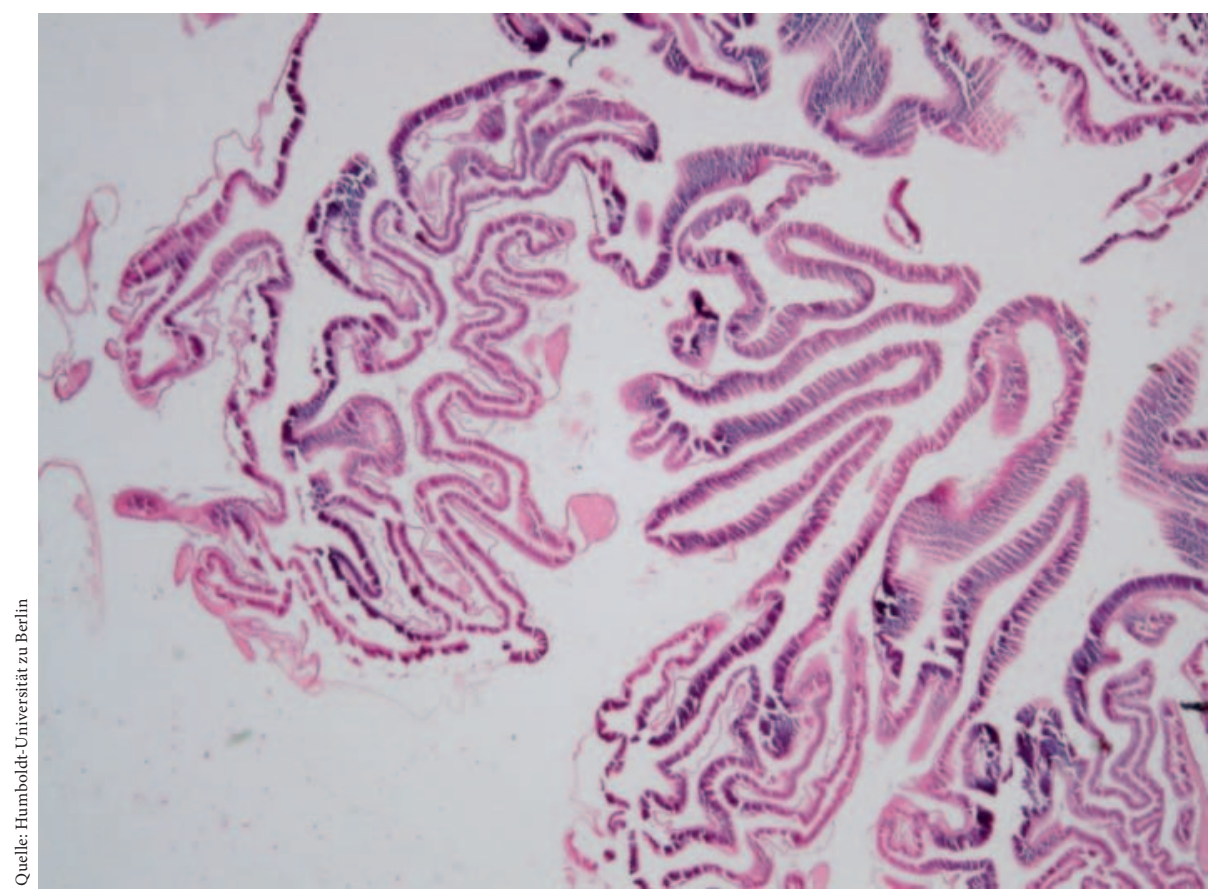

Abb. 1. Mikrotomschnitt von Magen und Divertikel eines Seesterns.

darm wird häufig ein beschleunigter, überbelasteter Alltag verantwortlich gemacht. Reinhard Saller allerdings mahnt bei solch einfachen Schlüssen zur Vorsicht. «Zu behaupten «Weil ich so viel Stress habe, habe ich jetzt einen Reizmagen` wäre zu einfach», sagt der Naturheilkundler. «Die Zusammenhänge sind um einiges komplizierter. Aber sie sind auf jeden Fall gegeben, und zwar in beide Richtungen.»

Ähnlich die Einschätzung von Pius Heer, Gastroenterologe in Olten: «Die Gleichung psychische Beeinträchtigungen ist gleich Magenbeschwerden wäre viel zu simpel», betont der Magen-Darm-Spezialist. Die Psyche sei nur einer von vielen Faktoren, die das vegetative Nervensystem beeinflussten, das seinerseits den Magen-DarmTrakt steure. "Andere Faktoren, die beim vegetativen Nervensystem mitspielen, sind zum Beispiel der Kaffeekonsum, Tag-Nacht-Rhythmen, die Jahreszeiten, die Temperatur oder das Volumen der zugeführten Nahrung.»

Bei rund zwei Drittel aller Patienten, die Pius Heer auf Magenbeschwerden hin untersucht, findet er keine organische Ursache. Diese Zahl der sog. funktionellen Störungen wird in der Literatur bestätigt. In vielen Fällen ist die positive Nachricht, dass nichts Krankhaftes vorliege, schon die Hälfte der Therapie, denn: «Die meisten Patienten fürchten sich vor einem Tumor», sagt Pius Heer. «Kann dieser ausgeschlossen werden, fällt auf einen Schlag die Angst ab, und die Patienten werden zugänglich für eine Behandlung.»

Heer nutzt oft das Vokabular aus der IT-Branche, um die Sachlage zu erklären: Als Schulmediziner müsse er analysieren, ob die «Hardware» reibungslos funktioniere. Sei dies sichergestellt, könne man bei der «Software» ansetzen. Eine derart gelagerte Störung sei für den Patienten zwar weniger bedrohlich als ein «Hardware»-Problem - ein funktionelles Reizdarmsyndrom zum Beispiel beeinträchtige die Lebenserwartung erwiesenermassen nicht -, schulmedizinisch aber sei die "Software» weit schwieriger zu beeinflussen. Der Grund: «Man kann gar nicht genügend betonen, was für ein hochkompliziertes, intelligentes Organ unser Magen-Darm-Trakt ist», sagt Heer. «Das ist physiologische Hightech und Biochemie im Höchstmass!
Hier müssen Millionen von chemischen Substanzen analysiert, Millionen von Gefahren gemeistert werden. Dagegen ist das Herz eine primitive Pumpe. Und das Herz mit der Liebe zu verbinden, ist geradezu vermessen, wenn man sich die Komplexität unseres Verdauungssystems einmal vor Augen hält.» Der Sitz der Gefühle, ist Pius Heer überzeugt, liegt nicht beim Herzen, sondern im Zentrum des Körpers.

Mit dieser Auffassung ist der Oltner Gastroenterologe beileibe nicht allein. Vielmehr ist der Bauch mit seinen Organen in den letzten Jahren vermehrt ins Interesse der Forschung gerückt, insbesondere bei der Neurologie: Man hat festgestellt, dass unsere Eingeweide von mehr als 100 Millionen Nervenzellen umhüllt sind. Das sind mehr Neuronen, als im gesamten Rückenmark zu finden sind. Manche Fachleute bezeichnen deshalb den Magen-Darm-Trakt als «zweites Gehirn» oder «Bauchhirn». Dieses «zweite Gehirn», so haben Wissenschafter herausgefunden, ist quasi ein Abbild des Kopfhirns - Zelltypen, Wirkstoffe und Rezeptoren sind exakt gleich.

Psychische Prozesse und das Verdauungssystem könnten weit inniger gekoppelt sein, als man bisher gedacht hat. "Das Bauchhirn spielt eine grosse Rolle bei Freud und Leid, doch die wenigsten Menschen wissen überhaupt, dass es existiert», sagte der US-Neurowissenschafter Michael Gershon, der als «Entdecker» des Bauchhirns gilt, gegenüber dem deutschen Geo Magazin. Was also ist das Bauchhirn?

Die Aufgabe des Bauchhirns besteht vor allem darin, die Verdauung zu steuern. Seine Besonderheit liegt darin, dass es unabhängig vom Kopfhirn viel weitreichendere Entscheidungen als einen Reflex bestimmen kann. Es denkt, fühlt und erinnert sich. Der «peristaltische Reflex» zum Beispiel, mit dem die Nahrung durch den Verdauungstrakt transportiert wird, wird völlig autonom vom Kopfhirn durch das Bauchhirn synchroni- 
siert. Dabei ist diese hochkomplexe Transportmaschine weit mehr als ein Reflex, sondern eine Art neurologische Kaskade: Rutscht eine Speise durch einen Darmabschnitt, werden Neurotransmitter ausgeschüttet, die wiederum andere Nervenzellen im Innern der Darmwand stimulieren. Diese senden hemmende und aktivierende Signale an Muskelzellen, die dann den wellenartigen Reflex ausführen.

Auch die Abwehrzellen an den Darmwänden sind grossenteils direkt mit dem Bauchhirn verbunden. Sie lernen, zwischen «gut» und «böse» zu unterscheiden. Diese Information wird gespeichert und bei Bedarf abgerufen. Wenn allerdings Gifte in den Körper gelangen, sendet das Bauchhirn Alarmsignale an das Kopfhirn. In Notsituationen soll der Kopf bereit sein, der Mensch sich seines Bauches bewusst werden und entsprechend reagieren - mit Erbrechen, Krämpfen, Durchfall. Ansonsten nimmt der Kopf die Botschaften meistens nicht bewusst wahr. Dabei verlaufen viel mehr Verbindungen, rund $90 \%$ gemäss jüngeren Forschungsergebnissen, von unten nach oben als umgekehrt. Die Signale von Bauch zu Hirn seien wichtiger als umgekehrt und «voller biologischer Bedeutung», lässt sich Michael Gershon zitieren.

«Das Darmhirn hat Macht», resümiert das Geo Magazin. Es könne die Daten seiner Sensoren selbst generieren und verarbeiten, und es kontrolliere ein Set von Reaktionen. Das Bauchhirn gibt den Nachbarorganen Anweisungen, koordiniert die Infektabwehr und die Muskelabwehr, und es muss schnell entscheiden und gespeichertes Wissen abrufen. Es ist funktionell organisiert und arbeitet mit Kreisläufen. Weiterhin ist es in der Lage, unterschiedliche Zustände zu registrieren und darauf zu reagieren. Das zweite Gehirn habe alles, was ein integratives Nervensystem brauche, kommt die Autorin zum Schluss. «Ja», gibt ein deutscher Kollege Gershons zu Protokoll, «man kann sagen, das Darmhirn denkt.»

Und es steht mit seiner grossen «Schwester», dem Kopfhirn, in ständiger Verbindung. Michael Gershon sagt, Bauch- und Kopfhirn sprächen die gleiche Sprache. Das hat auch Auswirkungen auf Krankheiten: Alzheimer- und Parkinson-Patienten beispielsweise weisen häufig ähnliche Gewebeschäden in Kopf und Bauch auf. Ausserdem: In der Tatsache, dass der Aufbau von Zelltypen und Wirkstoffen in Kopf- und Bauchhirn identisch ist, sehen viele Wissenschafter eine Erklärung dafür, weshalb psychiatrische Medikamente auch auf den Darm wirken - und umgekehrt. Ein Medikament, das gegen Reizdarm auf den Markt gebracht wurde, wurde ursprünglich als Anti-Angst-Droge entwickelt. Studien zeigen zudem, dass 40\% aller Reizdarm-Patienten an Angsterkrankungen und häufig auch an Depressionen leiden. Schwermut und Angst scheinen also aus dem Darm zu kommen, nicht umgekehrt.

In der Naturheilkunde sind diese Zusammenhänge empirisch schon lange bekannt. In vielen Fastentraditionen beispielsweise wird der Darm als Quelle des Glücks und der Gesundheit ebenso gepriesen, wie er als Gärbottich vieler Erkrankungen beargwöhnt wird. «Bis zum Aufkommen der modernen Psychopharmaka in den 1950er-Jahren wurde das breite Feld der Melancholie, wie Depressionen und depressive Verstimmungen damals genannt wurden, über den Magen-Darm-Trakt behandelt», erklärt Reinhard Saller. Zum Einsatz kamen hauptsächlich Bitterstoffe. Mit den synthetischen Psychopharmaka gerieten diese Erfahrungen weit in den Hintergrund, doch heute gewinnen sie an Bedeutung. Man weiss, dass Bittermittel in Wermutblättern, Enzianwurzel und Löwenzahnkraut therapeutisch gegen Ermüdungserscheinungen wirken, die Verdauung fördern, das Immunsystem stärken und zum Teil als natürliches Anti-

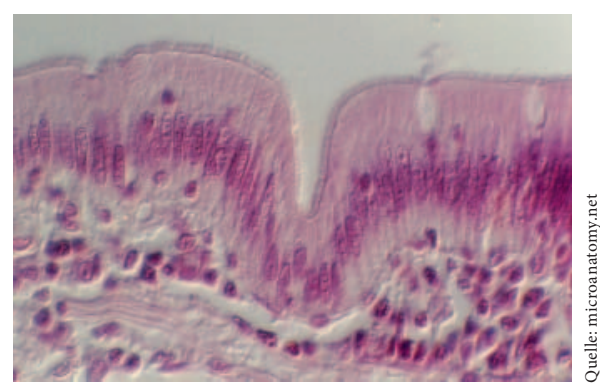

Abb. 2. Schnitt durch die Wand des Duodenums.

depressivum wirken. Auch die jüngeren Untersuchungen für die pflanzenbasierte (Mit-)Behandlung von Morbus Crohn zeigten als Nebeneffekt immer wieder antidepressive Wirkungen, die nicht direkt mit der Besserung des Magen-Darm-Befundes korreliert seien, sagt Reinhard Saller.

Kann man seine Befindlichkeit verbessern, indem man seinem Darm etwas Gutes tut? Ja, meint der Zürcher Naturheilkundler. Entscheidend sei das Essverhalten: Wie esse ich? Warum esse ich? Wie viel Zeit gestatte ich mir dafür? Hier lasse sich mit Gewürzen und «tausenderlei anderer Dinge» viel bewirken. (rfi)

\section{Literatur}

Burns AJ, Pachnis V: Development of the enteric nervous system: bringing together cells, signals and genes. Neurogastroenterol Motil 2009;21: 100-102.

Furness JB: The Enteric Nervous System. Oxford, Wiley-Blackwell, 2006.

Gershon MD: Functional anatomy of the enteric nervous system: a developmental perspective relevant to the pathogenesis of Hirschsprung's disease; in Holschneider AM, Puri P (eds): Hirschsprung's Disease and Allied Disorders. Amsterdam, Harwood Academic, 2000, pp 1958.

Grundy D, Schem M: Enteric nervous system. Curr Opin Gastroenterol 2007;23:121-126.

Laranjeira C, Pachnis V: Enteric nervous system development: recent progress and future challenges. Auton Neurosci 2009;151:61-69.

Luczak H: Neurologie - Wie der Bauch den Kopf bestimmt. Geo Magazin, 11/2000.

Spiller RC, Grundy D: Pathophysiology of the Enteric Nervous System: a Basis for Understanding Functional Diseases. Malden, Blackwell, 2004. 